\title{
Aerodynamic Performance of Biomimicry Snake-Shaped Airfoil
}

\author{
A. S. Mohd Rafie, A. M. Magaidi, O. F. Marzuki
}

\begin{abstract}
The cross-section shape and proportionality between geometrical dimensions are the most important design parameters of any lifting surfaces. These parameters affect the amount of the aerodynamic forces that will be generated. In this study, the focus is placed on the snake-cross-section airfoil known as the S-airfoil. It is found that there is a lack of available researches on S-airfoil despite its important characteristics. A parametric study on empty model of the S-airfoil with a cross-section shape that is inspired by the Chrysopelea paradise snake is conducted through numerical simulation. Simulation using 2D-ANSYS FLUENT17 software is used to generate the lift and drag forces to determine the performance of airfoil aerodynamic. Based on the results, the S-airfoil can be improved in performance of aerodynamic by reducing the thickness at certain range, whereby changing the thickness-to-chord ratio from 0.037 to 0.011 results in the increment of lift-to-drag ratio from 2.629 to 3.257. On other hand, increasing the height-to-chord ratio of the S-airfoil will increase maximum lift coefficient but drawback is a wide range of angles of attack regarding maximum lift-to-drag ratio. Encouraging results obtained in this study draws attention to the importance of expanding the research on S-airfoil and its usage, especially in wind energy.
\end{abstract}

Keywords: snake-cross-section airfoil, parametric study, CFD simulation, lift force coefficient, aerodynamic performance.

\section{INTRODUCTION}

Nature with its contents and phenomena is a prime source of inspiration to creativity and development. The flying snake locomotion could be considered as one of the most strikingly unique natural phenomena, which is distinguished from about 30 gliding animals in their capability to utilize their body as an aerodynamic surface without any appendages. In the available open literatures, it has been found that research studies related to the aerodynamics of gliding animals have been limited [1], despite of the advantages of these objects such as taxonomic diversity, large morphological, and evolutionary roots of gliding motion [2]-[3]. It has been pointed out that the

Revised Manuscript Received on December 30, 2019.

* Correspondence Author

A. S. Mohd Rafie*, Department of Aerospace Engineering, Universiti Putra Malaysia, Malaysia. Email: shakrine@upm.edu.my

A. M. Magaidi, Department of Aerospace Engineering, Universiti Putra Malaysia, Malaysia.

O. F. Marzuki, Department of Basic Science and Engineering, Universiti Putra Malaysia, Malaysia.

(C) The Authors. Published by Blue Eyes Intelligence Engineering and Sciences Publication (BEIESP). This is an open access article under the CC BY-NC-ND license (http://creativecommons.org/licenses/by-nc-nd/4.0/)
Chrysopelea paradise snake has a miraculous capability to change its flight characteristics by altering its glide speed and body size within Reynolds number $(R e)$ range between $1.5 \mathrm{x}$ $10^{3}$ and $5.0 \times 10^{3}$ [4]. During a glide trajectory, the snake experience Re increment as its thrust speed increased by six times from the starting takeoff speed [5].

Miklasz et al. carried out the first investigation regarding flying snake aerodynamic performance through body profiling [6]. The researchers substituted the Chrysopelea paradise snake's tubing sections shape by simple geometrical shape of half circle or filled arc. Moreover, this specie of the flying snake has efficient 4.5 glide ratios [7]-[8]. From the results of their study, the lift increased as the angle of attack (AOA) increased to $30^{\circ}$ and then the lift decreased slowly as AOA approach 1.5 maximum lift coefficient $\left(C_{L}\right)$. Furthermore, another study has used an anatomically accurate cross-section to produce a pattern in the lift performance at $\mathrm{AOA} 35^{\circ}$ for fluid flows with Re larger than $9.0 \times 10^{3}$ [2]. They found that the flying snake produce high maximum $\mathrm{C}_{\mathrm{L}}$ is 1.9 even though with the shaped and range of $\operatorname{Re}[6]$.

In view of the significant maximum $\mathrm{C}_{\mathrm{L}}$ observed in both abovementioned studies, there have been suggestions of its application as the lift-enhancement mechanism. The extra lift is speculated to be due to suction by vortices on the upper surface of the airfoil [9]. For the study presented in this paper, the primary aim is to understand the kinematic effects on an empty model of the Chrysopelea paradise snake cross section airfoil, also known as S-airfoil. This is done by changing the height and thickness of the airfoil in relative to its chord length, and the resultant aerodynamic performance of the airfoil is studied through the parametric study using two-dimensional Fluent ANSYS code.

\section{METHODOLOGY}

An empty cross section of S-airfoil has been chosen for the case study. Fig. 1 illustrates the schematic representation of an incompressible, unsteady flow past the S-airfoil. An artificial domain box is used as it is impossible to numerically replicate an unconfined flow. Following a previous study that has been presented in [5], the S-airfoil under study is positioned at the center of the square-shaped domain. For initial simulation studies, it is defined for air flow of velocity, $U$ is $5 \mathrm{~m} / \mathrm{s}$ passing a S-airfoil with chord length, $c=0.1898 \mathrm{~m}$, height, $h=0.0475 \mathrm{~m}$ and also thickness, $t=0.0045 \mathrm{~m}$. 


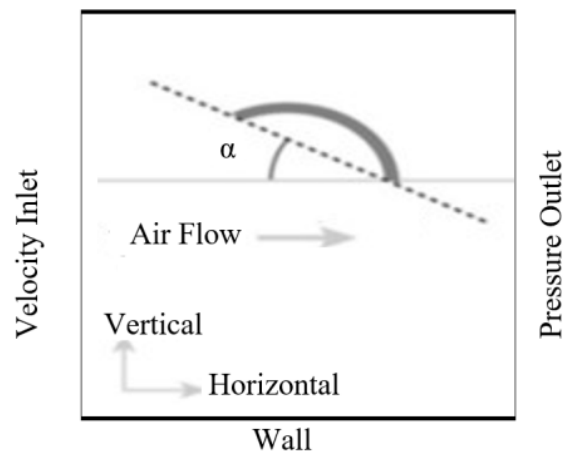

Fig. 1. Flow direction and AOA S-aifoil

Due to the effects of velocity, density, ambient air pressure, viscosity, length of airfoil and others, the flow passing on the surface of airfoil does not always have similar characteristics. Consequently, the forces and moments on the airfoil will also be different. These effects are represented by a non-dimensional parameter called the $R e$ [10], which is the inertia forces divided by viscous forces as shown by equation (1) where $U$ is airflow velocity, $v$ is air kinematic viscosity at $306.15^{\circ} \mathrm{K}$ and $L_{r e f}$ is chord length.

$$
R e=\left(U * L_{r e f}\right) / v
$$

To discretize the flow domain, the mesh model must be generated in order to solve governing equations of mass, momentum and energy. Through the grid independency test (GIT), the mesh influence on the lift force output results is studied. The studied is done with four types of mesh through 32 simulations. Fig. 2 shows the mesh is highly denser specifically around the airfoil and less than one for $\mathrm{y}^{+}$(wall) value to capture the complex flow structure with lower expected error [11]-[12].

In the meantime, the domain size is analyzed to scrutinize wake development around the S-airfoil. This is done in order to avoid the blockage phenomenon in the computational fluid dynamics (CFD) simulation. Based on the findings obtained from the analysis, it is found that the distance of the walls, which is the domain length and height can substantially affect the prediction for the performance of the geometrical airfoil cross section. The similar findings have also been reported by other researchers in [5] and [13]. In all, the airfoil is positioned at the center of the domain for the eight simulations that have been carried out for selected model of chord, height and thickness. The length of the domain edges, $L_{d o m}$ is selected in relative to the chord length. The output results are presented in Fig. 3 and it can be observed that, when $L_{d o m}$ is less than 40 times of the S-airfoil chord length, CFD simulation accuracy becomes more noticeable as the results for the generated lift force on the S-airfoil become inaccurate due to blockage effect. On the other hand, there is a small difference between the generated lift force values when $L_{d o m}$ is between 40 up to 70 times of the S-airfoil chord length. Hence, $L_{d o m}$ equals 40 times the chord length is taken as the optimum size for the domain by taking the simulation time into consideration.

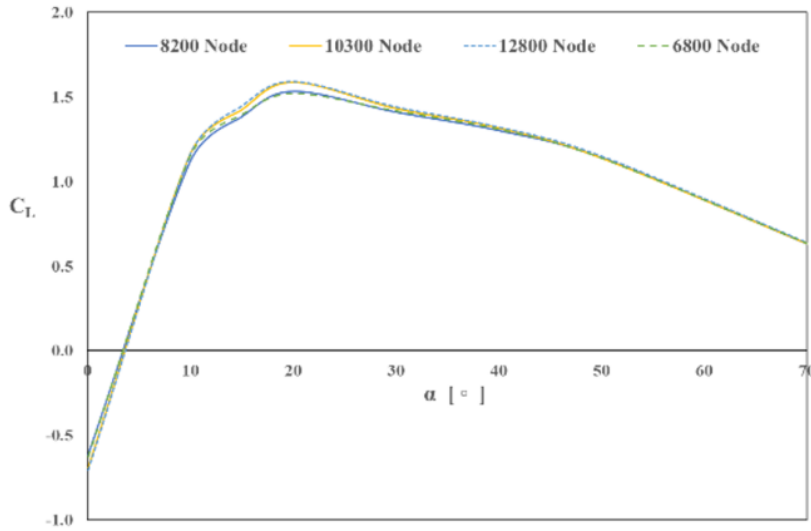

Fig. 2. Outputs from GIT simulation at $U=5 \mathrm{~m} / \mathrm{s}$

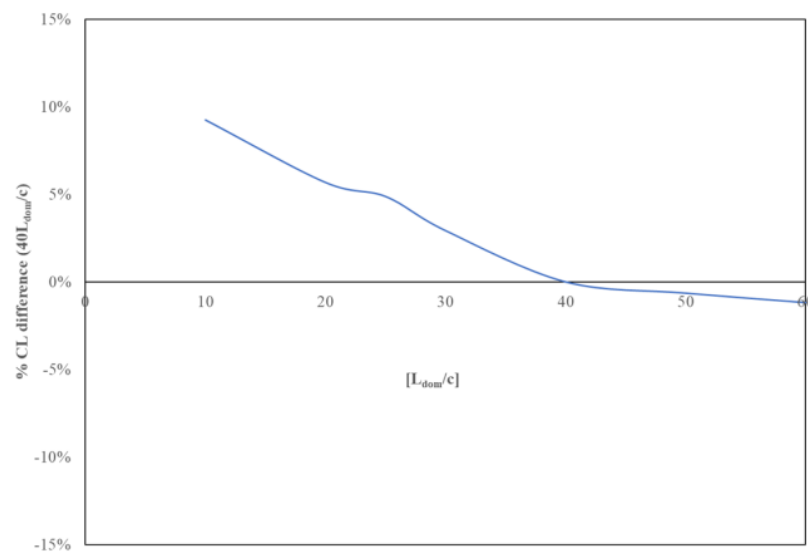

Fig. 3. Lift coefficient versus domain length over chord ratio

The model meshing is basically split into two zones: inner zone that is the $3^{*} c \times 3^{*} c$ block around the S-airfoil and zone away from the object known as outer zone. The inner zone is the repeated boundary layers over and throughout in the vicinity of the surface of the S-airfoil, so that the cells near to the airfoil is high to produce value $y^{+}<1$. As for outer zone, it has been further divided into four faces through four interior edges to achieve good structured mesh in order to accurately model the viscous forces. Table I presented some of the main mesh parameters while Fig. 4 shows the cut plane of the mesh within the domain.

Table-I: Main mesh parameters

\begin{tabular}{|c|c|}
\hline Parameter & Value \\
\hline \multicolumn{2}{|c|}{ Details of Mesh } \\
\hline Relevance Center & Fine \\
\hline Smoothing & High \\
\hline Element Minimum Size & $9.807 \times 10^{-1} \mathrm{~mm}$ \\
\hline Element Maximum Size & $9.807 \times 10^{1} \mathrm{~mm}$ \\
\hline Growth Rate & 1.2 \\
\hline \multicolumn{2}{|c|}{ Details of Inflation } \\
\hline Option & Maximum Thickness \\
\hline Number of Layers & 19 \\
\hline Growth Rate & 1.1 \\
\hline Total Thickness & $4.1 \mathrm{~mm}$ \\
\hline
\end{tabular}




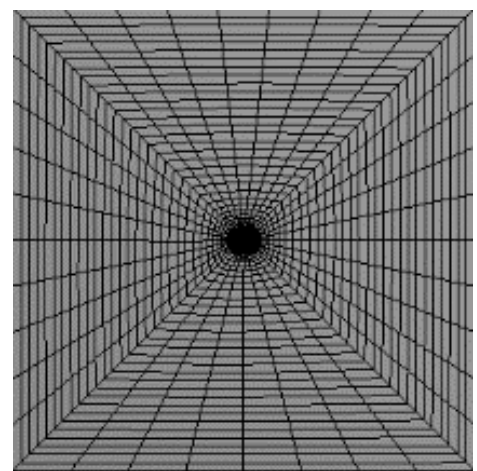

(a)

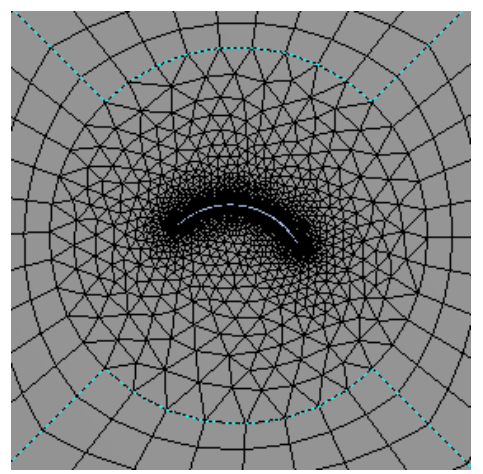

(b)

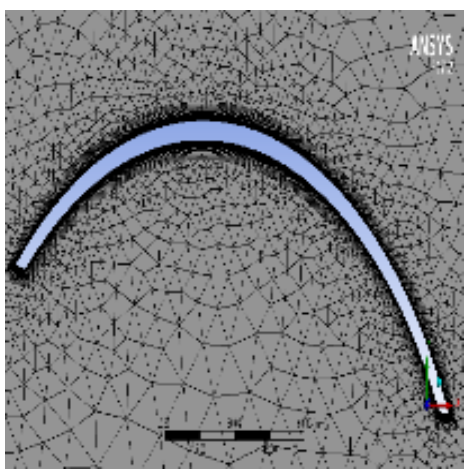

(c)

Fig. 4. CFD domain and meshing

The commercial CFD package Fluent Ansys17 is used for the numerical simulations in this study. The elliptic partial differential equations are solved through finite-volume method and the coupled iterative manner is used to solve the system of algebraic equations. The coupled pressure-velocity procedure updated the velocity and pressure fields together. Such approach has been known to be efficient in solving well-behaved problems and it can converge quicker than conventional segregated methods [5]. The implicit discretization of the mass flux and the pressure gradient terms in momentum equations is achieved through fully implicit coupling.

The high-quality mesh file that has been generated is inserted into the CFD solver. Before governing equations solve the mesh, the settings must be defined throughout the interface. In this case, the standard air characteristics at sea level have been selected to identify the boundary conditions. Furthermore, to accurately predict turbulent model for fluid flow characteristics around objects at high $R e$, the shear stress transport turbulent model, $k$ - $\omega$-SST is selected. This turbulent model has taken advantage of on the $k-\varepsilon$ model accuracy in the far-field region and $k-\omega$ model accuracy within the near-wall region. Such approach involves through merging a blending function between the two regions by converting the $k-\varepsilon$ model into the $k-\omega$ formulation, as explained in [1] and [9]. The overall solver settings used are summarized in Table II.

To validate the code for analyzing the empty model of the Chrysopelea paradise S-airfoil, a validation case study is done based on a previous study in [6] where the numerical and also experimental data is available for reference comparison. For this case study, the geometrical dimensions of the S-airfoil are defined through the scaling factor of 4.314 with respect to the tested model used in [6]. Moreover, the $R e$ for this simulation is set at $1.5 \times 10^{4}$. After the code has already been validated, a parametric investigation is conducted by varying the ratios of height-to-chord and thickness-to-chord of the empty S-airfoil. Table III highlights the different design settings applied in the parametric study. The documented peak aerodynamic performance is obtained through the simulation run for each range of AOA setting. By doing so, it provides an insight to the effects of the S-airfoil's aerodynamic performance by the change of its geometrical dimensions.
Table-II: Solver settings for numerical simulations

\begin{tabular}{|c|c|}
\hline \multicolumn{2}{|c|}{ Fluent Ansys17 Solver Setting } \\
\hline Type & Pressure Based \\
\hline Time & Transient \\
\hline Turbulence Model & k- $\omega$-SST \\
\hline Method & Pressure-Velocity Coupling \\
\hline Residual Error & $1.0 \times 10^{-6}$ \\
\hline Scheme & Coupled \\
\hline \multicolumn{2}{|c|}{ Spatial Discretization } \\
\hline Gradient & Green-Gauss Node Based \\
\hline Pressure & QUICK \\
\hline Momentum & QUICK \\
\hline Turbulent KE & QUICK \\
\hline Specific Dissipation & QUICK \\
\hline Time Step & $0.016 \mathrm{~s}$ \\
\hline \multicolumn{2}{|c|}{ Free Stream Conditions } \\
\hline Velocity & $5 \mathrm{~m} / \mathrm{s}$ \\
\hline Pressure & $101.325 \mathrm{KPa}$ \\
\hline Temperature & $288.15{ }^{\circ} \mathrm{K}$ \\
\hline
\end{tabular}

Table-III: Settings for the parametric investigation

\begin{tabular}{|c|c|c|c|c|c|}
\hline Model & $\boldsymbol{c}(\mathbf{m m})$ & $\boldsymbol{h}(\mathbf{m m})$ & $\boldsymbol{h} / \boldsymbol{c}$ & $\boldsymbol{t} \mathbf{( m m})$ & $\boldsymbol{t} / \boldsymbol{c}$ \\
\hline A & 189.80 & 47.50 & 0.25 & 4.50 & 0.0237 \\
\hline B & 189.80 & 47.50 & 0.25 & 2.00 & 0.0105 \\
\hline C & 189.80 & 47.50 & 0.25 & 7.00 & 0.0369 \\
\hline D & 189.80 & 28.47 & 0.15 & 4.50 & 0.0237 \\
\hline E & 189.80 & 66.43 & 0.35 & 4.50 & 0.0237 \\
\hline
\end{tabular}

\section{RESULTS AND DISCUSSION}

The results for the $C_{L}$ simulation of the S-airfoil in the validation case study are plotted against the AOA in Fig. 5. Through the results. it can be observed that the simulation results and the reference results in [6] are in a good agreement. Note that, there is a small discrepancy in the $C_{L}$ values at $\alpha=0^{\circ}$. The trend of $C_{L}$ value for the computed and the referenced is aligned quite well with each other. On the other hand, Fig. 6 shows the plot of the drag coefficient. Once again, the computed and the referenced results are in a great alignment with each other.

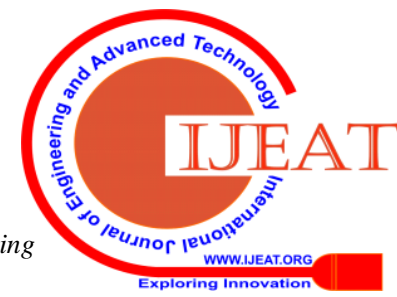




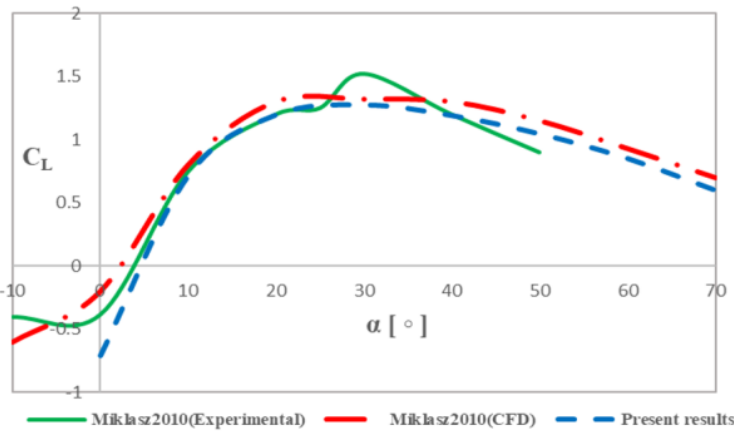

Fig. 5. Lift coefficient against angle of attack for validation case stuy

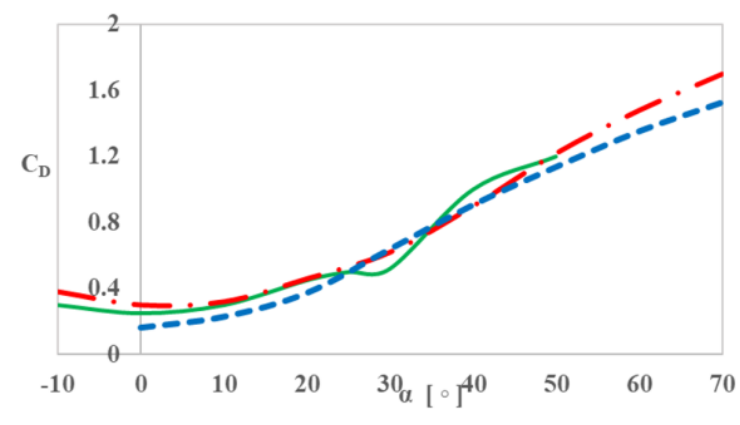

Fig. 6. Drag coefficient against angle of attack for validation case study

Based on these results for the validation case study, it can be taken the analysis code used has been validated and ready to be applied in the parametric investigation. To have a better understanding on the changes of aerodynamic characteristics for the S-airfoil with the changing of geometrical dimensions, the behavior is divided into two main regions of characteristic aerodynamics: pre- and post- stall regions. In this condition, the stall defines the AOA at which the maximum lift is reached.

Fig. 7 and Fig. 8 present the patterns of $C_{L}$ and drag coefficient $\left(C_{D}\right)$ for the S-airfoil models with different $t / c$ ratio. It can be seen that the flow patterns seem to behave similarly in the pre-stall region, indicating that both $C_{L}$ and $C_{D}$ are further increased within this region. The lift increased rate is found to be higher than that of drag in this pre-stall region. Meanwhile, in post-stall region, lift can be seen to decrease whereas drag continues to increase slightly more than in the pre-stall region. One of the benefits that can be observed from the effects of changing the S-airfoil thickness is the reduction of the range of near-maximum lift-to-drag ratio, accompanied with a slight increase in maximum $C_{L}$ value. By reducing the thickness from $7 \mathrm{~mm}$ to $2 \mathrm{~mm}$, the $C_{L}$ increases in average from 1.284 to 1.336 while $C_{D}$ reduces in average from 0.4884 to 0.4102 . In addition, maximum value of $C_{L}$ is risen from 1.4835 to 1.5060 by the same change in the S-airfoil thickness.

On the other hand, the effects of changing $h / c$ ratio for the S-airfoil can be observed from in Fig. 9 and Fig. 10. The flow patterns in both pre- and post-stall regions appears to have a similar trend to the ones observed in the effects of changing $t / c$ ratio. In general, the values of the lift-to-drag ratio and the maximum $C_{L}$ are improved by increasing $h / c$ ratio.

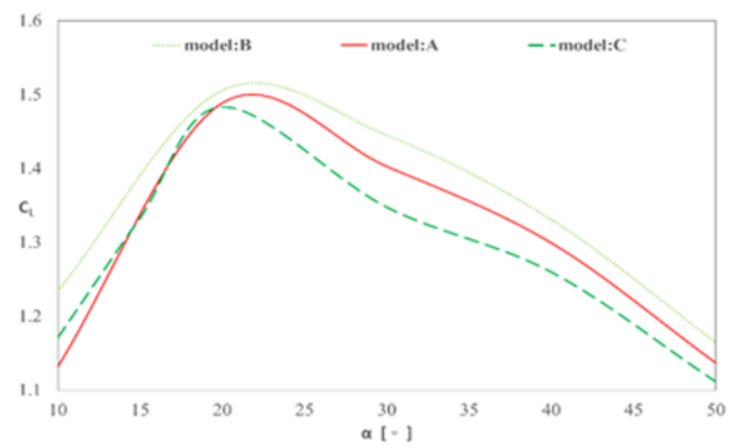

Fig. 7. Lift coefficient set against angle of attack for three S-airfoil models with different thickness at $R e=6.5 \times 10^{4}$

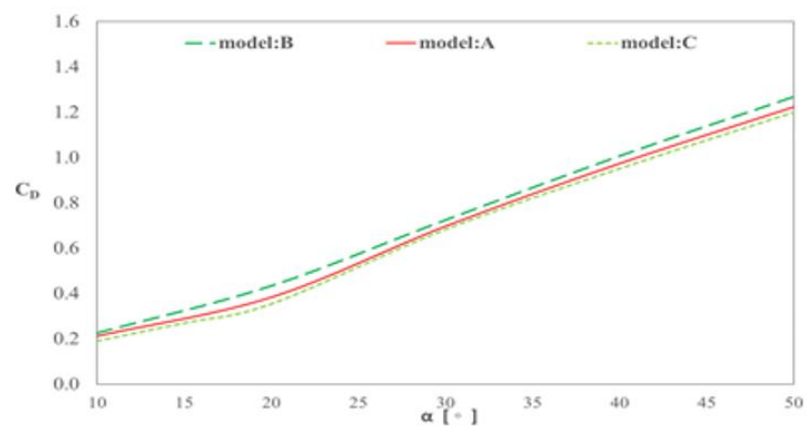

Fig. 8. Drag coefficient against angle of attack for three S-airfoil models with different thickness at $R e=6.5 \times 10^{4}$

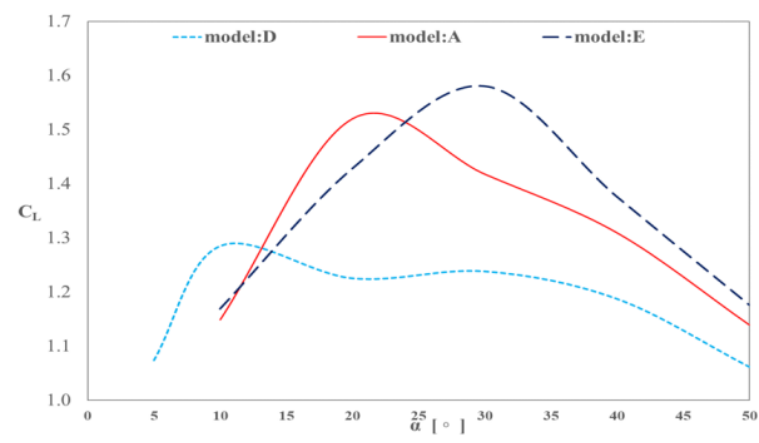

Fig. 9. Lift coefficient against angle of attack for three S-airfoil models with different height at $R e=6.5 \times 10^{4}$

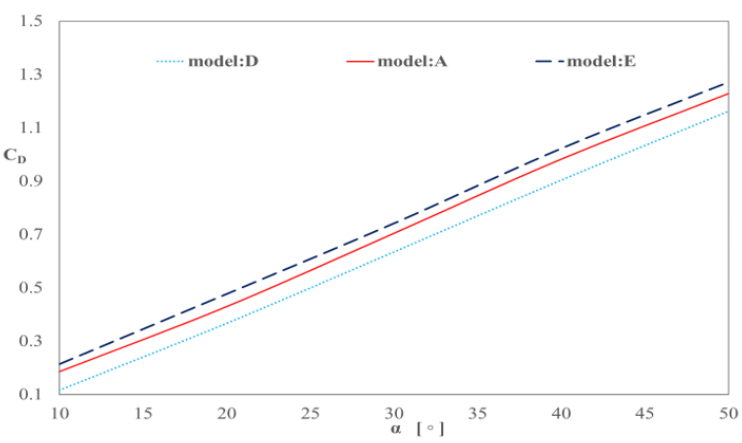

Fig. 10. Lift coefficient set against angle of attack for three S-airfoil models with different height at $\operatorname{Re}=6.5 \mathrm{X}$ 104 


\section{CONCLUSION}

In this research, a parametric study has been conducted on S-airfoil through CFD simulations using 2D Ansys Fluent17. The simulation analysis is done with conditions of free-stream flow at constant $R e=6.4 \times 10^{4}$. Five different S-airfoil models have been developed with different geometrical dimensions to study the impact of changing $t / c$ and $h / c$ ratios. Based on the results, it can be generally concluded that by reducing the $t / c$ ratio or increasing the $h / c$ ratio, the values of both maximum $C_{L}$ and lift-to-drag ratio will be increased. These results are in line with those reported in few previous studies.

\section{ACKNOWLEDGMENT}

The authors acknowledge the received financial support for this study from the Ministry of Education, Malaysia through fundamental research grant scheme (FRGS) with the project number FRGS/1/2018/TK09/UPM/02/2.

\section{REFERENCES}

1. F. Menter, "Two-equation eddy-viscosity turbulence models for engineering applications," AIAA Journal, vol. 32, 1994, pp. 1598-1605

2. R. Dudley, and S. Yanoviak, "Animal aloft: the origins of aerial behavior and flight," Integrative and Comparative Biology, vol. 51, 2011, pp. 926-936

3. R. Dudley, G. Byrnes, S. P. Yanoviak, B. Borrell, R. M. Brown, and J. McGuire, "Gliding and the functional origins of flight: biomechanical novelty or necessity?" Annual Review of Ecology, Evolution and Systematics, vol. 38, 2007

4. J. J. Socha, and M. LaBarbera, "Effects of size and behavior on aerial performance of two species of flying snakes (Chrysopelea)," Journal of Experimental Biology, vol. 208, 2005, pp. 1835-1847

5. S. Karabelas, B. Koumroglou, C. Argyropoulos, and N. Markatos, "High Reynolds number turbulent flow past a rotating cylinder," Applied Mathematical Modelling, vol. 36, 2012, pp. 379-398

6. K. Miklasz, M. LaBarbera, X. Chen, and J. J. Socha, "Effects of body cross-sectional shape on flying snake aerodynamics," Experimental Mechanics, vol. 50, 2010, pp. 1335-1348

7. J. J. Socha, "Kinematics: Gliding flight in the paradise tree snake," Nature, vol. 418, 2002

8. J. J. Socha, "Gliding flight in Chrysopelea: turning a snake into a wing," Integrative and Comparative Biology, vol. 15, 2011, pp. 969-982

9. D. Holden, J. Socha, N. Cardwell, and P. Vlachos, "Aerodynamics of the flying snake Chrysopelea paradisi: how a bluff body cross-sectional shape contributes to gliding performance," Journal of Experimental Biology, vol. 217, 2014, pp. 382-394

10. J. Anderson, Fundamentals of Aerodynamics, New York: McGraw-Hill Education, 2001

11. D. C. Wilcox, "Reassessment of the scale-determining equation for advanced turbulence models," AIAA Journal, vol. 26, 1988, pp. 1299-1310

12. W. W. Wood, "Boundary layers whose streamlines are closed," Journal of Fluid Mechanics, vol. 2, 1957, pp. 77-87

13. A. M. Mgaidi, A. S. Rafie, K. Ahmad, R. Zahari, M. A. Hamid, and O. F. Marzuki, "Numerical and experimental analyses of the flow around a rotating circular cylinder at subcritical regime of Reynolds number using $\mathrm{K}-\varepsilon$ and K- $\Omega$-SST turbulent models," ARPN Journal of Engineering and Applied Sciences, vol. 13, 2018

\section{AUTHORS PROFILE}

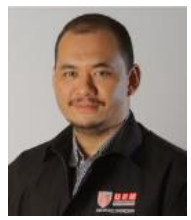

A. S. Mohd Rafie, is Associate Professor at Department of Aerospace Engineering, Universiti Putra Malaysia, Malaysia. He obtained his $\mathrm{PhD}$ in Aerospace Engineering from Universiti Putra Malaysia, Malaysia in 2007. He holds M. Eng (Mechanical), B. Eng. (Aeronautic) and Dip. Mech. Eng. (Aeronautic) from Universiti Teknologi Malaysia, Malaysia. He is currently the Vice Chairman of Aerospace Society Malaysia. His research interests include aerodynamics, aeroelasticity and green energy.

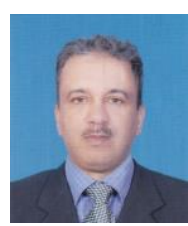

Amer M. Mgaidi, has graduated from Universiti Putra Malaysia (UPM), Malaysia in 2019 with a PhD degree in Aerospace Engineering. His fields of research interest are aerodynamics and green energy.

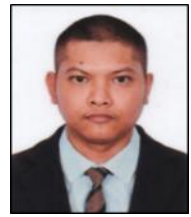

O. F. Marzuki, has received his $\mathrm{PhD}$ degree in Aerospace Engineering from Universiti Putra Malaysia, Malaysia in 2017. He was employed as an enumerator in Department of Aerospace Engineering, UPM. Currently, he is a senior lecturer at Universiti Putra Malaysia (Bintulu Campus). His field of research interests include aerodynamics and sustainable energy. 